\title{
Deep Learning based Cell Classification in Imaging Flow Cytometer
}

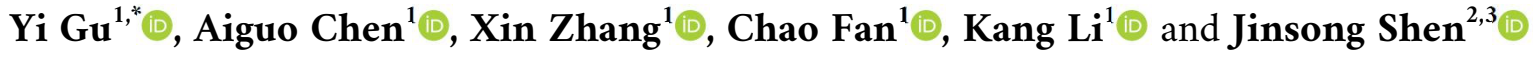 \\ 1. School of Artificial Intelligence and Computer Science, Jiangnan University, 214122, China \\ 2. Jiangsu Saideli Diagnostic Technology Co., Ltd, Jingiang, Jiangsu 214500, China \\ 3. Jiangsu Saideli Pharmaceutical Machinery Manufacturing Co., Ltd, Jingjiang, Jiangsu 214500, China \\ * Corresponding author: Yi Gu (8202101437@jiangnan.edu.cn)
}

Manuscript Revised 12 June 2021; Accepted 13 June 2021; Published 13 June 2021

Academic Editor: Xin Ning

Abstract: Deep learning is an idea technique for image classification. Imaging flow cytometer enables high throughput cell image acquisition and some have integrated with real-time cell sorting. The combination of deep learning and imaging flow cytometer has changed the landscape of high throughput cell analysis research. In this review, we focus on deep learning technologies applied in imaging flow cytometer for cell classification and real-time cell sorting. This article describes some recent research, challenges and future trend in this area.

Index Terms: Deep learning, Image classification, Neural network, Imaging flow cytometer

\section{Introduction}

Flow cytometer $[1,2]$ is an essential tool in high throughput single cell analysis, and has been widely applied in immunology research, cancer research, cell therapy, etc[3]. Imaging flow cytometer technology[4], which was invented in 2000s has become a game changer and greatly broadened the applications of flow cytometer.

Imaging flow cytometer (IFC) combines the imaging capability of microscope and high cell analysis throughout of flow cytometer. Thus IFC can acquire high resolution cell images at a throughput of hundreds cells to 10000 cells per second $[4,5]$. On the other hand, for each single cell, normally several image channels are acquired, which provides rich information that benefits cell analysis. For example, the Amnis imaging flow cytometer acquires bright field, dark field and up to 12 fluorescence channels[6]. Due to the high throughput of IFC, in each biology experiment, hundreds of thousands of multi-channel cell images are collected. Image processing and machine learning algorithms needs to be developed to analysis such large datasets. Also, image based cell sorting technologies[7] in microfluidics are developed recently, which require the algorithms to classify cell images in millisecond time lapse in order to support real-time image based cell sorting.

In order to automate image analysis and classification tasks in IFC, one approach is to use traditional image processing algorithm for cell image feature extraction, and use machine learning algorithm for classification. In 2016, Blasi, Thomas et al. published a label free cell cycle classification pipeline for IFC[8]. In this pipeline, cell image features are extracted using an opensource software (Cell Profiler[9]), and then RUSboosting is used for cell cycle classification. In 2019, Gu et al. developed a machine learning based cell classification algorithm and implemented it on FPGA platform to support high throughput real-time cell sorting[10]. In this work, cell image features are 
extracted by traditional image processing algorithm, then ROC curve is used for feature selection, and SVM is used for cell classification based on the selected image features. These cell classification algorithms are straight forward and easy to implement. Also, classification using traditional image features creates biological insights, such as how nucleus shape changes at different mitosis stages. However, some image feature extraction processes are time consuming thus not suitable for real-time image based cell sorting applications which requires short computation latency. On the other hand, features selection may require manual judgement, which relies on priori biology knowledge and experience.

Thanks to the development of deep learning[11], automated cell classification in IFC can be implemented using deep learning technique including deep neural network (DNN) [12] and convolutional neural network (CNN) [13]. A deep neural network (DNN) is a hierarchical network consists of an input layer, several hidden layers and an output layer for prediction, which is also known as a multilayer perception (MLP). The layers in a DNN are typically densely or fully connected. Convolutional neural network extracts image features using convolutional kernels followed by a pooling layer. And typically a fully connected layer follows the convolutional layers for image classification. The structure of CNN results in less trainable parameters and allows the network to be deeper.

Compared to cell image classification based on traditional image features, deep learning algorithms directly learning features from training datasets, thus skip the computational expensive image processing steps and the feature selection step which may require manual judgement. In addition, deep learning structures are suitable for transfer learning[14], which is idea for cell image classification, since even for the same cell type, the acquired cell images may vary from experiment to experiment due to different light excitation condition, flow condition and even cell staining condition.

Because of the advantages of deep learning, research focused on deep learning cell classification algorithms in IFC has sprung up recently. For example, Philipp Eulenberg et al. has designed a CNN network for label free cell cycle classification in 2017[15]. In 2018, Nao Nitta has used CNN to realize real-time image based cell sorting[16].
This article will be arranged as following: In section 2, mainstream IFC technologies will be briefly introduced; in section 3, recent research works on deep learning based cell classification in IFC are introduced, in section 4, challenges and future trends in this area is discussed.

\section{Image technologies in flow cytometer}

Mainstream imaging technologies in IFC can be cataloged into 2 main types: 1 . Multiplexed imaging devices (e.g. CCD) based technologies, 2. Single pixel photodetector based technologies. Mainstream imaging technologies in IFC will be briefly introduced in this section.

Imagestream is a typical multiplexed imaging device based IFC technology[17], which is also the earliest IFC technology. Imagestream adopts time delay integration (TDI) [18] method for IFC imaging. In TDI sensor design, rows of the sensor pixels shift their partial measurements to the adjacent rows synchronously with the motion of the moving cell images in order to capture very weak fluorescence cell images. There are also other types of multiplexed imaging device based IFC technologies such as multiple-field-of-view imaging flow cytometer (MIFC) [19], temporally coded excitation[20], etc.

Single pixel photodetector based technologies use single pixel photodetector (e.g. PMT) to measure light intensity in time-domain and then reconstruct cell images from timedomain signal. Due to the high sensitivity of photodetector, this type of method provides higher sensitivity and supports higher imaging throughput compared to multiplexed imaging device based method. However, this type of method requires specifically designed spatial to temporal transform mechanism and image reconstruction algorithms. Han et al. developed spatial-temporal transform method for image acquisition of fast moving cells[21]. This method uses a designed spatial mask that only transmits specific portion of cell image to transform cell image into time domain light intensity. And the image is reconstructed from the measured time domain signal. There are also other single pixel photodetector based methods developed for IFC such as radiofrequency-tagged emission (FIRE) [22], serial timeencoded amplified microscopy (STEAM)[23], etc. Recently, Han et al has developed a 3D imaging technology for IFC [24]. 
Table 1. summarizes the mainstream IFC technologies. In table 1, detector modality, imaging throughput, whether compatible with sorting function, and imaging modality is listed. As shown in Table 1, due to the imaging method and microfluidic design, some methods can be integrated with sorting function, which requires fast computation speed when designing classification algorithms.

Table 1. Summary of IFC technologies

\begin{tabular}{|c|c|c|c|c|}
\hline & Detector type & $\begin{array}{l}\text { Imaging } \\
\text { throughput }\end{array}$ & $\begin{array}{l}\text { Compatibility } \\
\text { with sorting }\end{array}$ & $\begin{array}{l}\text { Imaging } \\
\text { modality }\end{array}$ \\
\hline Imagestream & Multiplexed imaging device & $1000-3000$ & No & $\begin{array}{l}\text { Fluorescence; } \\
\text { Transmission; } \\
\text { Side-scatter }\end{array}$ \\
\hline MIFC & Multiplexed imaging device & $\sim 10000$ & No & Transmission \\
\hline Temporal coded excitation & Multiplexed imaging device & $\sim 2000$ & No & $\begin{array}{l}\text { Fluorescence; } \\
\text { Transmission }\end{array}$ \\
\hline Spatial temporal transform & Single pixel photodetector & $>1000$ & Yes & $\begin{array}{l}\text { Fluorescence; } \\
\text { Transmission; } \\
\text { Back-scattering }\end{array}$ \\
\hline FIRE & Single pixel photodetector & $\sim 50000$ & Yes & Fluorescence; \\
\hline STEAM & Single pixel photodetector & 10000 & Yes & $\begin{array}{l}\text { Fluorescence; } \\
\text { Transmission; }\end{array}$ \\
\hline 3D Imaging flow cytometer & Single pixel photodetector & $\sim 500$ & No & $\begin{array}{l}\text { Fluorescence; } \\
\text { Side-scattering }\end{array}$ \\
\hline
\end{tabular}

\section{Deep learning based cell image classification}

When designing deep learning cell image classification methods for IFC, the following four key elements need to be taken into consideration. First is the input of the algorithms. From the perspective of algorithms input, classification methods can be cataloged into 2 types: 1 . Classify cells using cell images; 2. Classify cells directly using time domain signal measured by single pixel photodetector. As shown in Fig.1, compared to type 1, type 2 algorithms skip the image reconstruction step thus is more time efficient. Second is image dimension, there are algorithms developed for $2 \mathrm{D}$ imaging flow cytometer and 3D imaging flow cytometer respectively. Third is whether the classification needs to be integrated with real-time cell sorting capability since realtime cell sorting requires short computational latency. Usually, latency under $10 \mathrm{~ms} / \mathrm{s}$ cell is needed for efficient and accurate sorting, since certain cell flowing speed is required to maintain a stable flow condition. Fourth is the imaging method. The classification algorithms described in the article are developed for different imaging methods as shown in Table.1, thus the algorithms have to fit specific imaging method.

In this section, we will describe representative works of 4 typical catalogs of deep learning based cell classification in IFC: 1. Cell classification based on 2D images; 2. Cell classification directly using un-reconstructed waveforms; 3 . Cell classification using 3D images; 4. Cell classification for real-time image activated cell sorting.
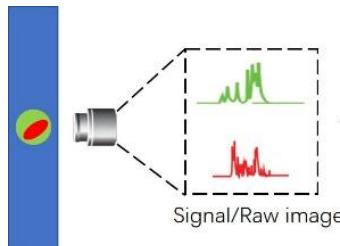

Reconstruction

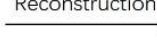

(a)
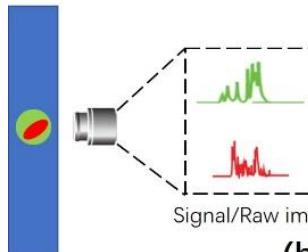

(b)

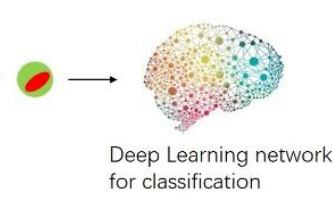
for classification
Fig.1. (a) Cell classification using reconstructed images. (b) Cell classification directly using time domain signal. 


\subsection{Label-free cell cycle classification using deep CNN}

When classifying cells at different mitosis phases[25], the conventional and widely used approach is using conventional intensity based flow cytometer to analyze fluorescence intensity, which requires specific protein markers and staining process. The staining process is not only tedious and label intensive, but also brings unexpected cell property changes. Thanks to the invention of IFC, researchers are able to acquire large amount of cell images, which makes high-throughput label-free cell analysis possible. In 2017, Philipp Eulenberg et al developed a deep learning pipeline for label free cell cycle analysis[15]. In this work, Imagestream method is used to acquire cell images. Due to the readout mechanism of TDI imaging sensor, the image reconstruction is automatically done by the hardware readout process thus no image reconstruction algorithm is needed and the cell classification pipeline can directly use the cell images as input. However, due to the property of Imagestream method, image quality is very sensitive to any slight fluidic disturbance, so this pipeline can only be used for cell classification and can not integrate with real-time cell sorting module.

As shown in fig.2(a), only bright field and dark field images are needed for classification thus no fluorescence image is needed. In this work, fluorescence images are used for training sample labeling.

The CNN network architecture is illustrated in fig.2(a). First, the bright field and dark filed images are combined into a 2-channel input. The network consists of 42 layers, it starts from 3 dual-path reduction modules, followed by 10 normal dual-path modules, one global average pooling layer, one fully connected layer and a softmax layer. In addition, tdistributed stochastic neighbor embedding (tSNE) is used for feature visualization. Since mitosis is a continuous biology process, tSNE shows a continuous change of deeps learning extracted features over the whole mitosis process. A dual-path modules consists of a convolution layer, a batch normalization layer and an activation layer. The architecture of dual-path modules is depicted in fig.2(b), where (i) is a normal dual-path module and (ii) is a dual-path reduction module. As mentioned above, the first three dual-path reduction modules reduce the original input dimension significantly.

The experiment results in this work shows that the deep CNN architecture is able to classify full 7 mitosis phases classification only using label free images. Remarkably, this work demonstrated that CNN can classify G1,S,G2 phases which are extremely hard to distinguish. Also, the results show that deep learning method outperforms traditional image feature based method in cell cycle classification.

In addition, authors also demonstrated the classification of disease progression using $\mathrm{CNN}$ in this work, and mentioned that this architecture not only works in label free cases but can also be extended to fluorescence cell image classification.

(a)
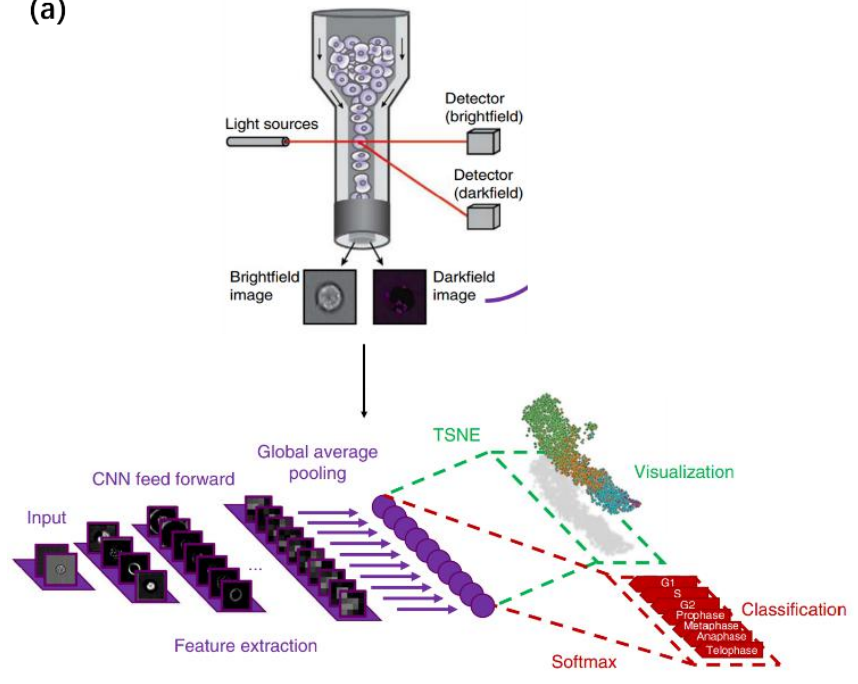

(i)

(ii)
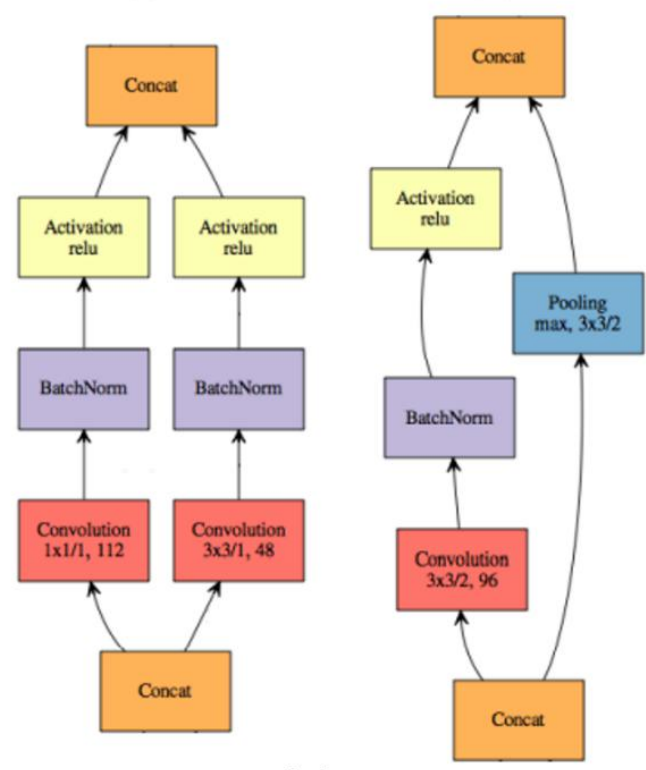

(b)

Fig.2. (a) Illustration of cell classification pipeline; (b) (i) normal dual-path module (ii) dual-path reduction module

3.2 Cell classification directly using time series signal in STEAM IFC 
As shown in section 2, most IFC technologies requires sophisticated cell image reconstruction. The reconstruction step is time consuming. Cell classification directly using measured signal skips the image reconstruction step and shorten the whole classification time latency[26,27]. In 2019, Yueqin $\mathrm{Li}$ et al developed a CNN based cell classification pipeline directly using measured time series signal as input[27].

The data acquisition is using STEAM IFC which is shown in Table.1, so the acquired data is a one dimensional time series signal detected by ultrafast analog-to-digital-converter (ADC).

Data preparation is shown in fig.3(a), each waveform containing measurements of multiple cells is divided into 100 waveform elements with $50 \%$ overlap ratio. Each of the 100 waveforms contain information of one single cell or nothing. To fit the CNN architecture, the waveforms are reshaped into 2 dimension, with one dimension corresponds to the laser pulses, one dimension corresponds to the sampling points in each laser pulse. To improve memory efficiency and shorten processing time, the reshaped waveform is reduced by a reduction factor of 40 in the first dimension. Then the preprocessed waveforms are shuffled and grouped into batches for CNN training. The dataset is labeled into 3 catalogs: SW-480 colorectal cancer cells, OTII hybridoma white blood cells and blank examples (no cell). The CNN architecture is shown in fig.3(b). The architecture consists of 16 convolutional layers with $3 \times 3$ kernel size stride of 1 , and ReLU. 3 max pooling layers with window size of $2 \times 2$ are used for down sampling. Convolutional layers are followed by 3 fully connected layers, and dropout regularization is applied to each fully connected layer. The fully connected hidden layers are activated by ReLU. Finally, a softmax layer is used to generate predictions.

In this work, authors also benchmarked the computation speed of the classification pipeline using different hardware, and the lowest time latency is achieved using a NVIDIA P100 GPU. The time latency is $3.6 \mathrm{~ms}$ per cell, which indicates that this pipeline is a possible solution for real-time cell sorting.

3.3 CNN for label free cell classification in 3D sidescattering IFC
As shown in Table.1, most mainstream IFC technologies are limited to $2 \mathrm{D}$ imaging, thus cell classification is based on $2 \mathrm{D}$ images. In 2020, Rui Tang et al developed a deep learning cell classification pipeline using 3D cell images[28]. The algorithm is developed for the 3D IFC technology developed by the same research group in 2019 , and is the first work of 3D deep learning cell classification in IFC[24].

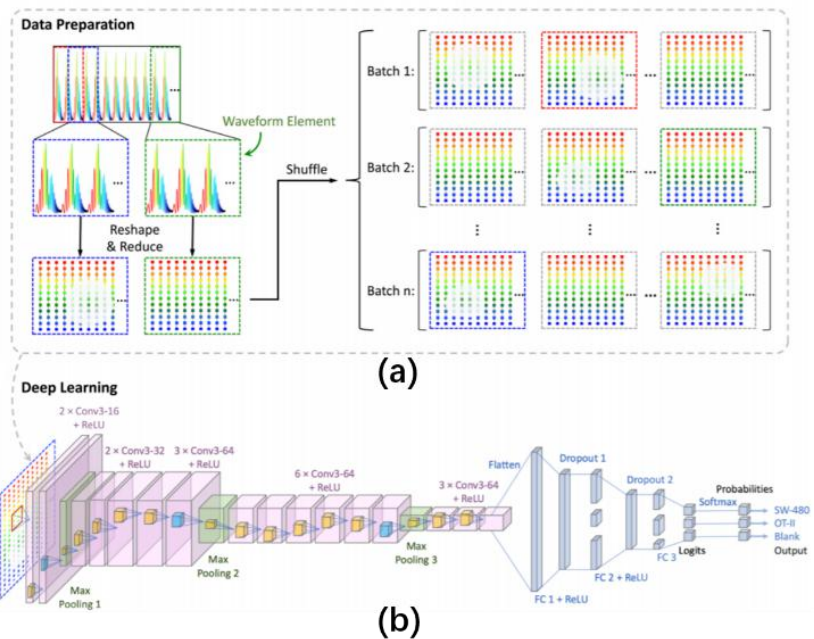

Fig.3. (a) Data preparation process; (b) Illustration of the CNN architecture

The corresponding IFC technology is illustrated in fig.4(a), cells flowing through the microfluidic channel are scanned by light sheet and spatial pin holes, more details of the hardware design are described in []. Then time series signal is measures by single pixel photodetector, and reconstructed into 3D images. And deep learning is used for cell classification based on $3 \mathrm{D}$ side-scattering images.

In this work, authors developed 2 Unet[29] based architectures: (1) 2DCNN architecture as shown in fig.4(b)(i); (2) 3DCNN architecture as shown in fig.4(b) (ii). In the $2 \mathrm{DCNN}$ architecture, the $3 \mathrm{D}$ images are sliced along the depth direction and the 2D slices are treated as multi-channel input, 2D convolution kernels are used in this architecture as in a typical Unet. In the 3DCNN architecture, 3D images are directly used as input, and $3 \times 3 \times 3$ convolution kernels are used. In both architectures, a fully connected layer and a softmax layer are connected to the latent space to generate classification predictions. In both architectures, loss function is the summation of prediction cross-entropy loss and mean-square-error loss.

In this work, authors used the following 5 architectures to classify MCF-7 cells, Hela cells, HEK-293 cells, and compared the performance of the 5 architectures: 1 . Use $2 \mathrm{D}$ 
projection as input and Resnet18 architecture as classification model; 2. Use 3D image as input and Resnet1 8 as classification model; 3 . Use $2 \mathrm{D}$ projection as input and the proposed 2DCNN as classification model; 4. Use 3D images as input and the proposed 2DCNN as classification model; 5. Use 3D images as input and the proposed 3DCNN as classification model. And based on the experiment results, the 3DCNN architecture achieved the best classification performance.

Although this work developed the first 3D deep learning cell classification model, it can not be integrated with realtime cell sorting due to the property of the corresponding IFC technology.

\subsection{Intelligent Image-Activated Cell Sorting}

Cell sorting technology separates specific cell group from cell mixture, which is widely used in rare cell enrichment, single cell sequencing, etc. [30-32] Conventional cell sorting technology sort cells based on scattering/fluorescence intensity. The IFC technology makes image activated cell sorting possible. However, to achieve image activated cell sorting, the classification computation latency needs to be lower than $10 \mathrm{~ms} /$ cell.

In 2018, Nao Nitta et al demonstrated the first image activated cell sorting experiments using deep learning algorithm [33].

The whole work flow is shown in fig.5(a). First, FIRE IFC technology is used for multi-fluorescence-channel cell image acquisition. Second, cell images are reconstructed from the measured time series signals. Third, deep learning algorithm is used for real-time cell classification. Fourth, once a cell is classified as in the target group, the Dualmembrane push-pull cell sorter is activated to deflect the target cell into the collection microfluidic channel.

A CNN architecture shown in fig.5(b) is used to classify platelet aggregate, leukocyte and single platelet. The input is a $105 \times 168$ image, and the CNN architecture consists of 6 convolution layers with max pooling and dropout. Then follows 2 fully connected layers to generate prediction. The total processing latency is under $32 \mathrm{~ms} / \mathrm{cell}$ on GPU platform, and 100 cells/s processing rate is achieved by adopting pipeline processing.
3D imaging flow cytometry
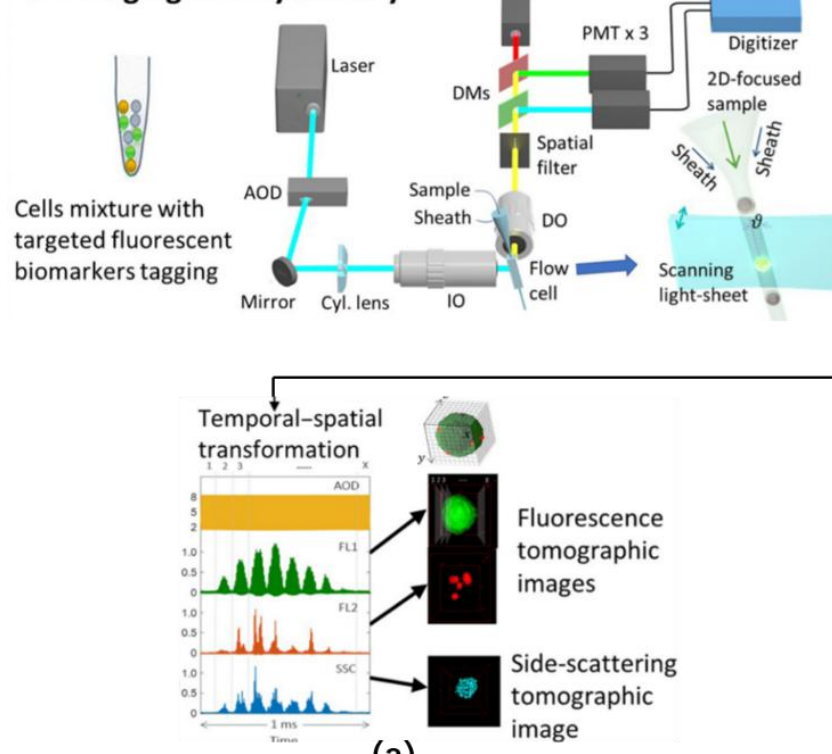

(a)
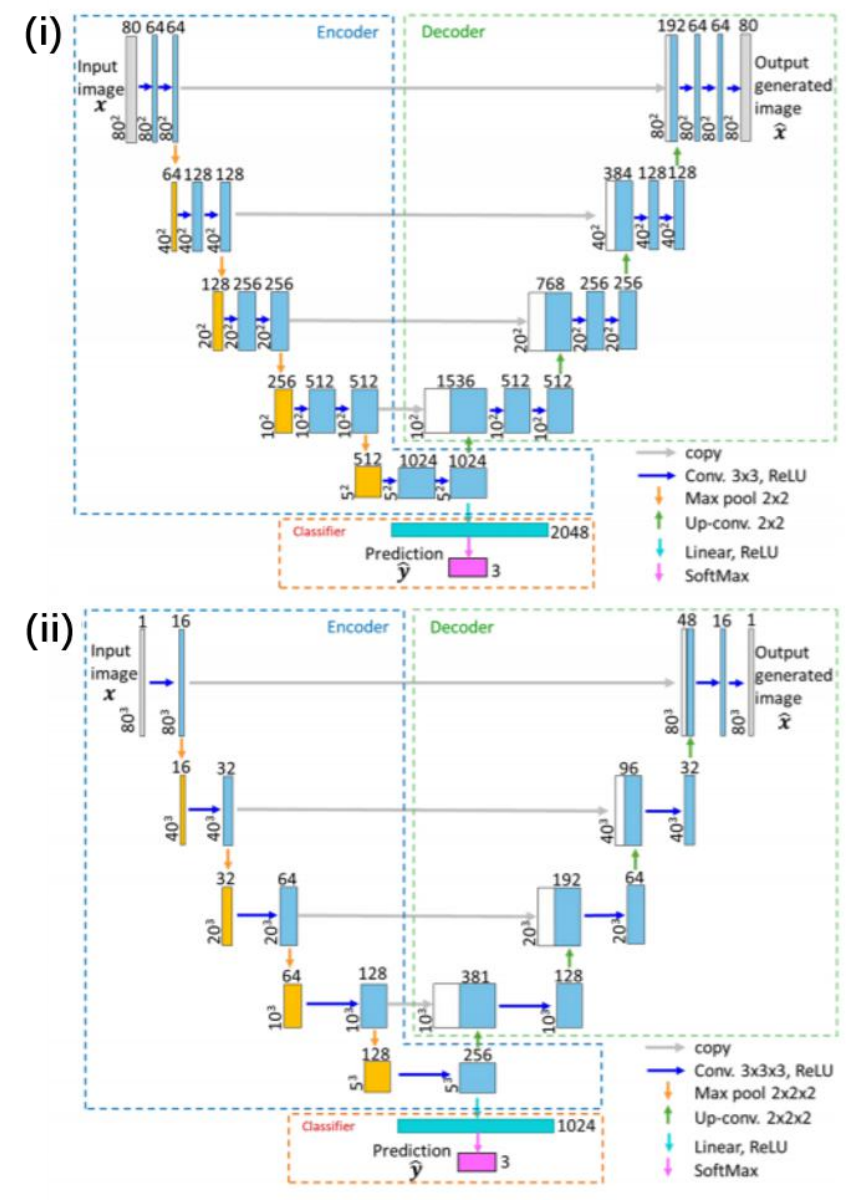

(b)

Fig.4. (a) The 3D IFC hardware system; (b) (i) 2DCNN architecture, (ii) 3DCDNN architecture

In this work, sorting of $3 \mu \mathrm{m}$ and $6 \mu \mathrm{m}$ beads is also demonstrated using a slightly modified CNN structure, where 4 convolution layers are used instead of 6. Also, by using more advanced processor and data transfer hardware, 


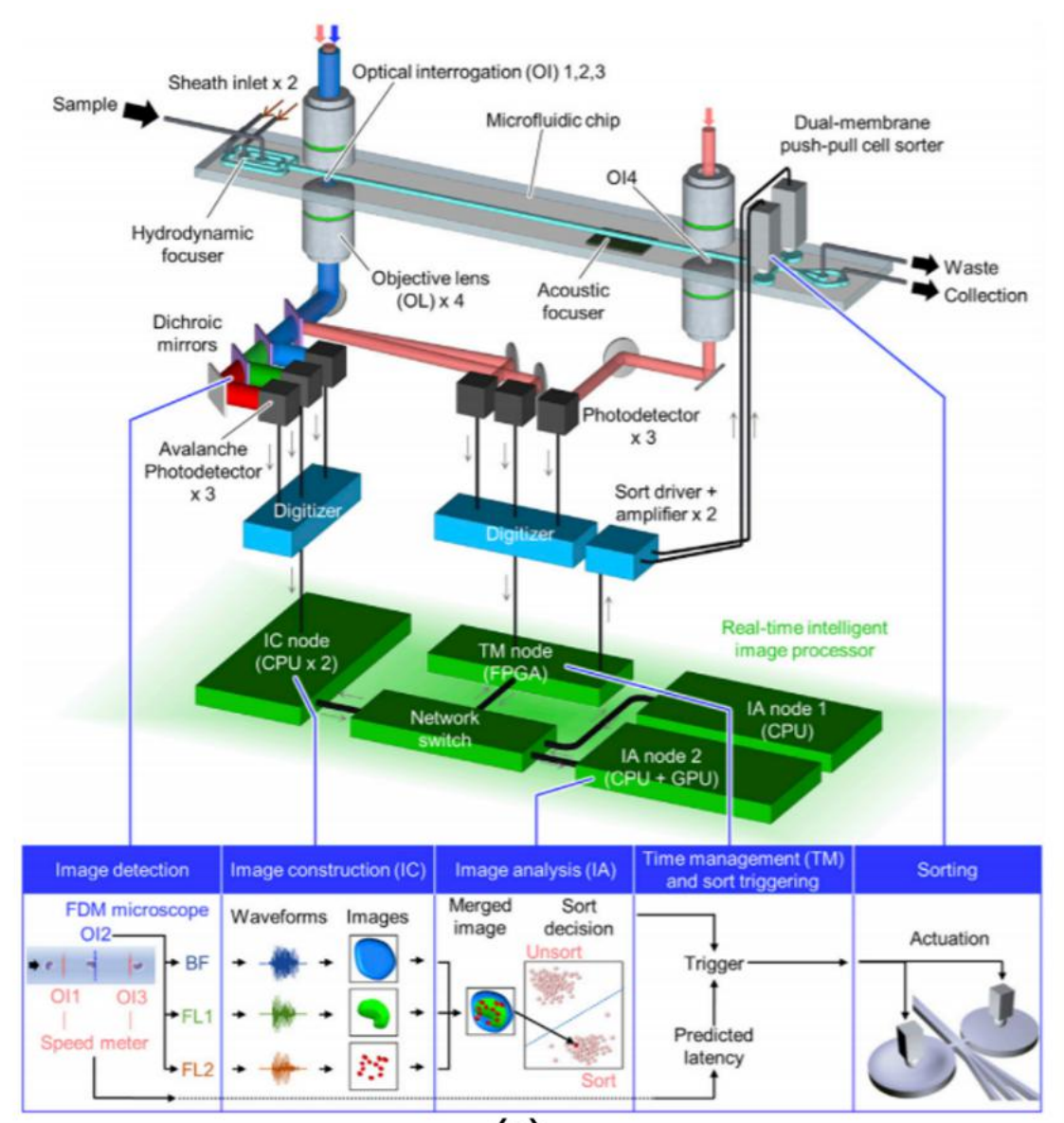

(a)

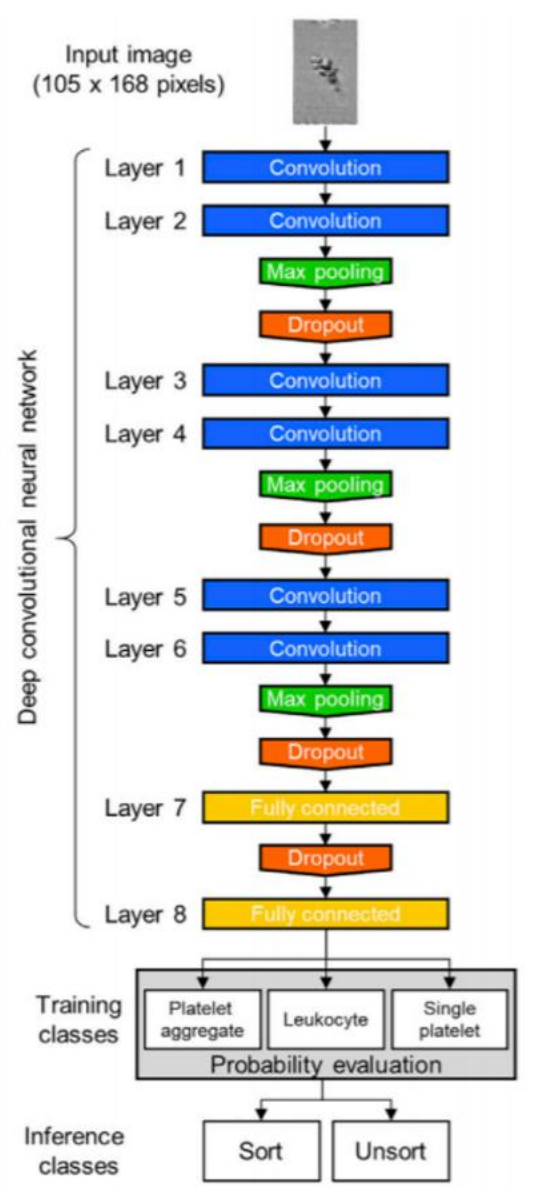

(b)

Fig.5. (a) Whole work flow of image activated cell sorting; (b) Architecture of CNN for cell classification

this research group has improved the sorting throughout to $>1000$ cells $/ s[34]$.

\section{Challenges and future trends}

Current challenges in deep learning based classification in IFC can be summarized as follows:

(1) Due to occlusion, blurry by focal depth and lack of zaxis resolution, 2D images is not sufficient for many classification applications. Although Rui Tang et al have developed 3D IFC and corresponding deep learning algorithm, the imaging resolution and throughput are still limited. This challenge requires development of both IFC imaging technology and deep learning classification algorithms.

(2) Most classification algorithms in IFC are supervised methods, thus labels need to be generated for training samples. Researchers may use several pure cell subgroups as training dataset[28], meaning each IFC run only contains cells from one specific group. However, this is not practical in most real biology applications, because we always need to classify different cell types in a cell mixture. Another method for labeling is to use additional fluorescence staining[15], which may cause unexpected cell property change and also requires extra labor. Furthermore, if cells can be differentiated by staining, there's no need for IFC. Actually, in many applications, fluorescence intensity is not sufficient for classification, which creates the demand of IFC and corresponding deep learning algorithms. Manual labeling is a possible solution, but the labeling process is labor intensive, particularly in IFC applications, because when cell type is changed, sometimes, researchers have to redo labeling process. In semi-supervised or selfsupervised learning, only few labeled training samples are needed. Given the fact that IFC is easy to acquire large number of un-labeled data, and the labeling process is labor intensive, semi-supervised or selfsupervised learning is a promising solution $[35,36]$.

(3) Image activated cell sorting is a milestone in IFC cell classification. However, it's still limited to 2D imaging. 3D image activated cell sorting will be the next major milestone in the field of IFC cell classification. This 
requires the development of fast 3D image classification algorithms as well as 3D IFC technology compatible with sorting device.

\section{Conclusion}

IFC technologies enables high throughput single cell imaging which generates massive amount of data. Deep learning is an idea approach for cell classification in IFC. Furthermore, deep learning automates the cell classification in IFC and enables real-time image activated cell sorting. This article reviews recent progress in the field of deep learning based cell classification in IFC, and summarizes the challenges and future trends.

\section{Acknowledgement}

This work was supported in part by the National Natural Science Foundation of China under Grants U20A20228 and 61772241

\section{References}

[1] Bonner, W. A., Hulett, H. R., Sweet, R. G., \& Herzenberg, L. A. (1972). Fluorescence activated cell sorting. Review of Scientific Instruments, 43(3), 404-409. Schmidhuber, J. (2012, June).

[2] Picot, J., Guerin, C. L., Le Van Kim, C., \& Boulanger, C. M. (2012). Flow cytometry: retrospective, fundamentals and recent instrumentation. Cytotechnology, 64(2), 109-130.

[3] Laerum, O. D., \& Farsund, T. (1981). Clinical application of flow cytometry: a review. Cytometry: The Journal of the International Society for Analytical Cytology, 2(1), 1-13.

[4] Han, Y., Gu, Y., Zhang, A. C., \& Lo, Y. H. (2016). Imaging technologies for flow cytometry. Lab on a Chip, 16(24), 4639-4647.

[5] Goda, K., Filby, A., \& Nitta, N. (2019). In flow cytometry, image is everything.

[6] Basiji, D. A. (2016). Principles of Amnis imaging flow cytometry. In Imaging Flow Cytometry (pp. 13-21). Humana Press, New York, NY.

[7] LaBelle, C. A., Massaro, A., Cortés-Llanos, B., Sims, C. E., \& Allbritton, N. L. (2020). Image-Based Live Cell Sorting. Trends in Biotechnology.

[8] Blasi, T., Hennig, H., Summers, H. D., Theis, F. J., Cerveira, J., Patterson, J. O., ... \& Rees, P. (2016). Label-free cell cycle analysis for high-throughput imaging flow cytometry. Nature communications, 7 (1), 1-9.

[9] Carpenter, A. E., Jones, T. R., Lamprecht, M. R., Clarke, C., Kang, I. H., Friman, O., ... \& Sabatini, D. M. (2006). CellProfiler: image analysis software for identifying and quantifying cell phenotypes. Genome biology, 7(10), 1-11.

[10] Gu, Y., Zhang, A. C., Han, Y., Li, J., Chen, C., \& Lo, Y. H. (2019). Machine learning based real-time image-guided cell sorting and classification. Cytometry Part A, 95(5), 499-509.

[11] LeCun, Y., Bengio, Y., \& Hinton, G. (2015). Deep learning. nature, 521(7553), 436-444.

[12] Sharma, P., \& Singh, A. (2017, July). Era of deep neural networks: A review. In 2017 8th International Conference on Computing, Communication and Networking Technologies (ICCCNT) (pp. 1-5). IEEE.

[13] Rawat, W., \& Wang, Z. (2017). Deep convolutional neural networks for image classification: A comprehensive review. Neural computation, 29(9), 2352-2449.

[14] Pan, S. J., \& Yang, Q. (2009). A survey on transfer learning. IEEE Transactions on knowledge and data engineering, 22(10), 1345-1359.

[15] Eulenberg, P., Köhler, N., Blasi, T., Filby, A., Carpenter, A. E., Rees, P., ... \& Wolf, F. A. (2017). Reconstructing cell cycle and disease progression using deep learning. Nature communications, 8(1), 1-6.

[16] Nitta, N., Sugimura, T., Isozaki, A., Mikami, H., Hiraki, K., Sakuma, S., ... \& Goda, K. (2018). Intelligent image-activated cell sorting. Cell, 175(1), 266-276.

[17] Zuba-Surma, E. K., Kucia, M., Abdel-Latif, A., Lillard, J. W., \& Ratajczak, M. Z. (2007). The ImageStream System: a key step to a new era in imaging. Folia histochemica et cytobiologica, 45(4), 279-290.

[18] Lepage, G., Bogaerts, J., \& Meynants, G. (2009). Time-delayintegration architectures in CMOS image sensors. IEEE Transactions on Electron Devices, 56(11), 2524-2533.

[19] Schonbrun, E., Gorthi, S. S., \& Schaak, D. (2012). Microfabricated multiple field of view imaging flow cytometry. Lab on a Chip, 12(2), 268-273.

[20] Gorthi, S. S., Schaak, D., \& Schonbrun, E. (2013). Fluorescence imaging of flowing cells using a temporally coded excitation. Optics express, 21(4), 5164-5170.

[21] Han, Y., \& Lo, Y. H. (2015). Imaging cells in flow cytometer using spatial-temporal transformation. Scientific reports, 5(1), $1-10$.

[22] Diebold, E. D., Buckley, B. W., Gossett, D. R., \& Jalali, B. (2013). Digitally synthesized beat frequency multiplexing for 
sub-millisecond fluorescence microscopy. Nature Photonics, 7(10), 806-810.

[23] Goda, K., Ayazi, A., Gossett, D. R., Sadasivam, J., Lonappan, C. K., Sollier, E., ... \& Jalali, B. (2012). High-throughput singlemicroparticle imaging flow analyzer. Proceedings of the National Academy of Sciences, 109 (29), 11630-11635.

[24] Han, Y., Tang, R., Gu, Y., Zhang, A. C., Cai, W., Castor, V., ... \& Lo, Y. H. (2019). Cameraless high-throughput threedimensional imaging flow cytometry. Optica, 6(10), 12971304.

[25] Schafer, K. A. (1998). The cell cycle: a review. Veterinary pathology, 35(6), 461-478.

[26] Ota, S., Horisaki, R., Kawamura, Y., Ugawa, M., Sato, I., Hashimoto, K., ... \& Noji, H. (2018). Ghost cytometry. Science, 360(6394), 1246-1251.

[27] Li, Y., Mahjoubfar, A., Chen, C. L., Niazi, K. R., Pei, L., \& Jalali, B. (2019). Deep cytometry: deep learning with realtime inference in cell sorting and flow cytometry. Scientific reports, 9(1), 1-12.

[28] Tang, R., Zhang, Z., Chen, X., Waller, L., Zhang, A. C., Chen, J., ... \& Lo, Y. H. (2020). 3D side-scattering imaging flow cytometer and convolutional neural network for label-free cell analysis. APL Photonics, 5(12), 126105.

[29] Ronneberger, O., Fischer, P., \& Brox, T. (2015, October). Unet: Convolutional networks for biomedical image segmentation. In International Conference on Medical image computing and computer-assisted intervention (pp. 234-241). Springer, Cham.

[30] Cho, S. H., Chen, C. H., Tsai, F. S., Godin, J. M., \& Lo, Y. H. (2010). Human mammalian cell sorting using a highly integrated micro-fabricated fluorescence-activated cell sorter ( $\mu$ FACS). Lab on a Chip, 10(12), 1567-1573.

[31] Rato, S., Golumbeanu, M., Telenti, A., \& Ciuffi, A. (2017). Exploring viral infection using single-cell sequencing. Virus research, 239, 55-68.

[32] Mattanovich, D., \& Borth, N. (2006). Applications of cell sorting in biotechnology. Microbial cell factories, 5(1), 1-11.

[33] Nitta, N., Sugimura, T., Isozaki, A., Mikami, H., Hiraki, K., Sakuma, S., ... \& Goda, K. (2018). Intelligent image-activated cell sorting. Cell, 175(1), 266-276.

[34] Isozaki, A., Mikami, H., Tezuka, H., Matsumura, H., Huang, K., Akamine, M., ... \& Goda, K. (2020). Intelligent imageactivated cell sorting 2.0. Lab on a Chip, 20(13), 2263-2273.

[35] Zhou, X., \& Belkin, M. (2014). Semi-supervised learning. In Academic Press Library in Signal Processing (Vol. 1, pp. 1239-1269). Elsevier.
[36] Jing, L., \& Tian, Y. (2020). Self-supervised visual feature learning with deep neural networks: A survey. IEEE Transactions on Pattern Analysis and Machine Intelligence.

\section{Biographies}

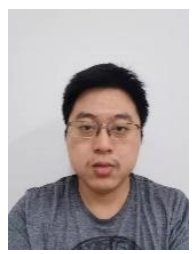

Yi Gu received the B.S degree in information Engineering from Shanghai Jiao Tong University, Shanghai, China, in 2012, and the M.S degree in Applied Physics from Cornell University, US, in 2014, and Ph.D degree in Electrical Engineering from University of California San Diego, US, in 2019. He is currently an Assistant Professor with the School of Artificial Intelligence and Computer Science, Jiangnan University. His current research interests include bioinformatics, artificial intelligence, image processing and their applications. (8202101437@jiangnan.edu.cn)

Aiguo Chen received the B.S degree in Computer Science and Technology from China University of Mining and Technology, Xuzhou, China, in 1998, and the M.S degree in Light Industry Technology

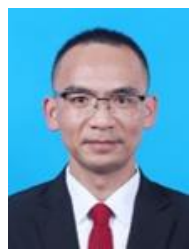
and Engineering from Jiangnan University, Wuxi, China, in 2007, and Ph.D degree in Light Industry Information Technolog from Jiangnan University, Wuxi, China, in 2017. He is currently an Assistant Professor with the School of Artificial Intelligence and Computer Science, Jiangnan University. His current research interests include machine learning, artificial intelligence, image processing and their applications. (agchen@jiangnan.edu.cn)

Xin Zhang received the Ph.D. degree in South China University of Technology, China. She is currently a lecturer in the School of Artificial Intelligence and Computer Science, Jiangnan University, China. Her research interests include evolutionary computation and their applications in intelligent manufacturing. (zhangxin@jiangnan.edu.cn)

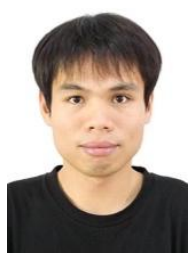

Chao Fan received a M. S degree in Informatics from Peking University, Beijing, China, in 2012, and the Ph.D degree in Engineering from the University of Tokyo, Japan, in 2018. He is currently an Assistant Professor with the School of 
Artificial Intelligence and Computer Science, Jiangnan University. His research interests involve artificial intelligence, network science, and image processing. (fanchao@jiangnan.edu.cn)

Kang $\mathbf{L i}$ received the B.S degree in Computer science and technology from Northeastern University, Shenyang, China, in 2018. He is currently pursuing the M.D. degree with School of Artificial Intelligence and Computer Science, Jiangnan University. His research area includes computational intelligence, image processing and their applications on medicine. (zslygdsx@gmail.com)

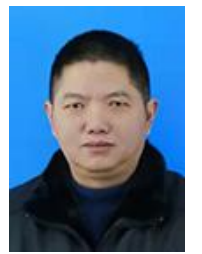

Jinsong Shen received the B.S degree from Tongji University, Shanghai, China. He is currently a CEO of Jiangsu Saideli Diagnostic Technology Co., Ltd. (e-mail: Jinsongshen2021@163.com) 